demonstrated that the dog brain maintains this activity for several hours after complete decapitation.

This work was supported by grant $B 2185$ from the National Institute of Neurological Diseases and Blindness.

David D. Gilbot

Willtam W. Cotanch

MORRIS B. GLOVER

Division of Nourological Surgery,

University of Wisconsin,

Madison, Wisconsin.

${ }^{1}$ Bouckaert, J. J., and Jourdan, F., Arch. Int. Pharmacodyn., 53, 523 (1936).

2Chute, A. L., and Smyth, D. H., Quart. J. Exp. Physiol., 29, 379 (1939).

'Geiger, A., and Magnes, J., Amer. J. Physiol., 143, 33 (1945).

- White, R. J., Albin, M. S., and Verdura, J., Science, 141, 1060 (1963).

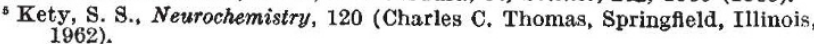

\section{Functional Location of Adrenergic Receptors on Blood Vessels in Muscle}

THE functional location of $\alpha$ - and $\beta$-adrenergic receptors in muscle vessels is uncertain ${ }^{1}$; they might be situated on resistances in series, in parallel or at the same point.

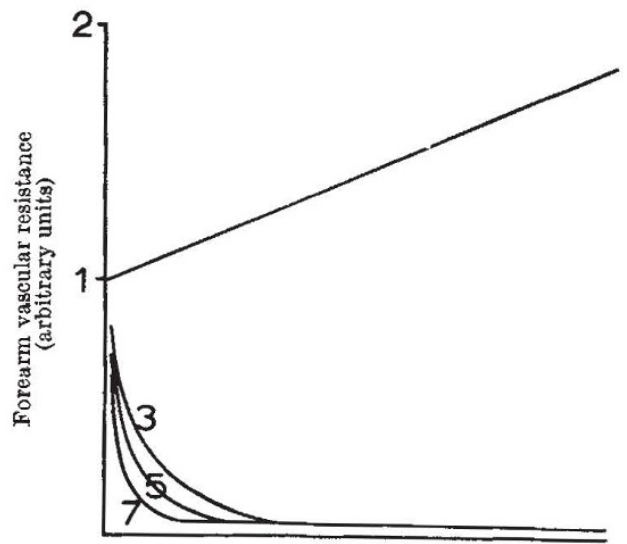

Blood catccholamine concentration

Fig. 1. relationship between calculated blood concentration of catecholamines and forearm vascular resistance. The upper straight line is obtained during intra-arterial infusion of noradrenaline or of adrenaline after blockade of the $\beta$-receptors. The lower family of curves is obtained with isoprenaline or adrenaline after blockade of the $\alpha$-receptors ; the figures on these curves show the duration of the infusion in minutes

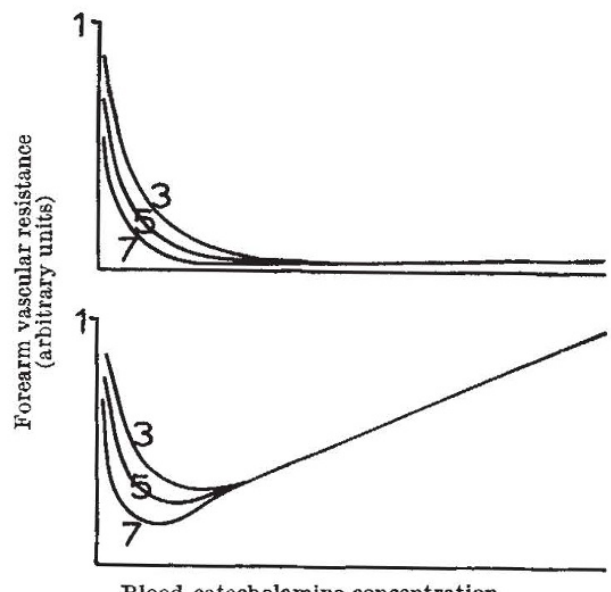

Fig. 2. Theoretical dose response curves obtained from the curves shown in Fig. 1 if the $\alpha$ - and $\beta$-receptors were stimulated simultaneously by infusion" of adrenaline, and were located on resistance in parallel (above) or in series (below)

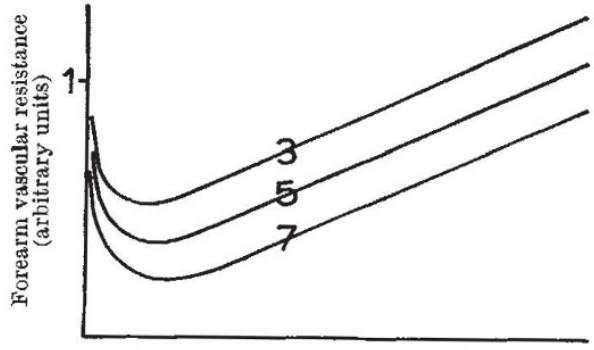

Blood catecholamine concentration

Fig. 3. Actual shape of dose response curves for adrenaline or for a mimic the response to adrenaline

In the human forearm the effect of $\alpha$-adrenergic stimulation is a square wave constrictor response which does not change with $\operatorname{tim} \vartheta^{2}$. The effect of $\beta$-adrenergic stimulation is more complex, with a sustained dilatation which increases progressively with time. Dose response curves for the separate $\alpha$-and $\beta$-adrenergic effects are illustrated in Fig. 1.

Using these separate dose response curves it is possible to construct models of the dose response curves which should be obtained if the $\alpha$ - and $\beta$-receptors were stimulated simultaneously, and were situated on resistances in series or in parallel (Fig. 2). The assumptions made in deriving these curves are: (1) That the shape of dose response curve for $\alpha$-adrenergic effects in forearm muscle does not differ substantially from the curve for the fore. arm as a whole. (2) That the parallel resistances in muscle behave in a homogeneous way. These theoretical curves in Fig. 2 differ from the actual dose response curves obtained experimentally (Fig. 3). A curve of the type shown in Fig. 3 can only be explained on the assumption that the $\alpha$-and $\beta$-receptors in muscle are situated neither in series nor in parallel, but on the same resistance.

Medical Unit,

R. D. Lowe

St. George's Hospital Medical School, London, S.W.1.

${ }^{1}$ Ahlquist, R. P., Amer. J. Physiol., 153, 586 (1948),

2 Lowe, R. D., and Robinson, B. F., Clin. Sci., 26, 81 (1964).

\section{Effects of Sugars on Intestinal Transfer of Amino-acids}

Ir is well established that there are specific processes for the active transfer of both sugars and amino-acids by the small intestine. Since these substances can be transferred against a concentration gradient and since this transfer is prevented by metabolic inhibitors, energy derived from metabolism is presumably utilized in the transfer mechanisms. It would seem, therefore, that the mechanisms for sugar and amino-acid transfers might share some step in the overall absorption process even if it was only the linkage with a common energy source. Experiments were carried out to investigate this possibility.

Everted sacs (approximately $17 \mathrm{~cm}$ long) of intestine were made from the middle fifth of the combined jejunum and ileum of the rat. The sacs were filled with $1 \mathrm{ml}$. bicarbonate saline and were suspended in $50 \mathrm{ml}$. bicarbonate saline to which various sugars and amino-acids were added. At the end of a $30-\mathrm{min}$ incubation period at $38^{\circ} \mathrm{C}$, the amount of amino-acid (glycine or methionine) transferred from the mucosal fluid was determined.

The results obtained with glycine are shown in Table 1. The two actively transferred sugars had quite different effects on glycine transfer, glucose causing stimulation and galactose causing inhibition. Both these effects were abolished by phlorrhizin, which in the absence of 